\title{
Morphologische und ökologische Anpassungen von Acabaria-Arten (Octocorallia) im Roten Meer an verschiedene Formen der Wasserbewegung ${ }^{*}$
}

\author{
H. SCHUHMACHER \\ Rubr-Universität Bochum, Lebrstubl für Spezielle Zoologie; \\ Bocbum, Bundesrepublik Deutschland
}

\begin{abstract}
Morphological and ecological adaptations of Acabaria species (Octocorallia) to different kinds of water movement in the Red Sea. The influence of turbulent current in comparison to regularly unidimensionally flowing current on the gorgonians Acabaria pulcbra, A. erytbraea and A. biserialis has been studied in the merchant harbour of Eilat. Their distribution on the concrete pillars supporting the harbour pier was restricted to shadow areas $(2-0.5 \%$ of the illumination above the free surface). Only in the surf-influenced surface layer with oscillating and pendular currents of changing intensity and direction (down to $2 \mathrm{~m}$ ), $A$. pulchrat and the very similar but rare species $A$. erythraea were found. Corresponding to these findings, $A$. pulchra could be observed in the natural reef only in the dark channels and crevices of the reef edge where currents are irregular according to the varying surf intensity. In contrast, $A$. biserialis settled on harbour pillars below the $A$. pulchra region in a zone of a regularly unidimensionally flowing long shore current. The rigid colonies of $A$. pulchra are small (up to $6 \mathrm{~cm}$ high) and thus capable of resisting surf pressure. They are branched in all directions; the polyps are extended on all sides of the branches. A. biserialis reached a hight of $20 \mathrm{~cm}$ at the harbour pillars and $30 \mathrm{~cm}$ at the slope base of various reefs. Colonies were always two-dimensionally branched. Fans grew from the lateral sides of the harbour pillars into the prevailing long shore current. Corresponding conditions were found on the reef. Perpendicularly positioned to the current, they achieve a maximum filter effect. The polyps of $A$. biserialis are situated in two lateral rows along the branches. The different growth forms of $A$. pulchra und $A$. biserialis seem to be species specific. Both species exclude themselves in their distributional patterns, depending on water movement: $A$. pulchra (together with $A$. erytbraea) is the single gorgonian in the Red Sea, settling on the reef edge and outer flat exposed to surf influence; $A$. biserialis grows in deeper water, like a typical sea fan, in the zone of unidimensionally flowing long reef current.
\end{abstract}

\section{EINLEITUNG}

Untersuchungen zur Zonierung von Korallenriffen liegen aus allen Meeresgebieten vor (vgl. Stoddart 1969 u. a.). Sie beschreiben eine unterschiedliche Verteilung von

* Mit Unterstützung der Deutschen Forschungsgemeinschaft. 
Korallen und anderen benthischen Organismen im Riff zwischen Ufer und Riffhang bzw. tieferem Vorriff. Über die Gründe, warum eine Art hier vorkommt oder dort fehlt, können sie jedoch höchstens Vermutungen anstellen. Denn in einem gut ausgebildeten Riff ist es normalerweise nicht möglich, unter der Vielfalt der sich gegenseitig beeinflussenden ökologischen Fakstoren, die gleichzeitig auf die in Frage stehende Art einwirken, die entscheidenden Parameter in ihrem Wirkungsgrad sicher zu erkennen.

Eine Möglichkeit, in einer Faktorenanalyse die spezifischen ökologischen Ansprüche einer Art näher zu präzisieren, bietet die experimentelle Änderung eines Umwelteinflusses bei gleichzeitiger Invarianz der übrigen Bedingungen. Bei Eilat (Golf von Aqaba, Israel) wurde im Rahmen von Langzeitbeobachtungen (von Dezember 1969 bis März 1971) an verschiedenen Kunstbauten (Hafenanlagen) die benthische Besiedlung in Abhängigkeit der wichtigsten riffökologischen Parameter, wie Wasseraustausch, Sedimentationsrate und Lichtexposition, studiert. Die einzelnen Untersuchungsplätze hierfür waren so ausgewählt, daß sich nur ein oder höchstens zwei Faktoren wesentlich änderten, die anderen Umweltbedingungen dagegen ungefähr konstant waren. Der Einfluß der Helligkeit auf die Verbreitung von Rifforganismen konnte z. B. eindeutig an der "Lichtorgel Hafenpfeiler" nachgewiesen werden (SchuHMacher 1973). Die ebenfalls auf den Stützpfeilern des Handelshafens siedelnden Gorgonarien Acabaria pulcbra und $A$. biserialis zeigten neben der Abhängigkeit von einem gewissen Helligkeitsgrad auch eine jeweils spezifische Anpassung an unterschiedliche Formen der Wasserbewegung, wie im folgenden erörtert werden soll.

Die Lage des Untersuchungsgebietes, die Pier des Handelshafens von Eilat, wurde schon ausführlich charakterisiert (SCHUHMAcher 1973), so daß hier außer einer Schilderung der Strömungsverhältnisse die übrigen Gegebenheiten nur kurz wiederholt werden sollen. Der Landekai erstreckt sich von NNO nach SSW und wird in einer Länge von ca. $550 \mathrm{~m}$ und einer Breite von ungefähr $12 \mathrm{~m}$ von acht Reihen Stützpfeilern aus Beton getragen. Die äußerste Pfeilerreihe steht $12 \mathrm{~m}$ tief im Wasser, die ufernächste (achte Reihe) nur $1 \mathrm{~m}$ tief. Die Pieranlage war zu Beginn der Untersuchungen sechs Jahre alt.

Hinsichtlich der Strömungsverhältnisse im Golf von Aqaba fehlen noch langjährige Meßreihen, um eindeutige Aussagen über Gesetzmäßigkeiten machen zu können. Nur bei den unmittelbar dem Wind unterworfenen Oberflächenwellen lassen sich die vorherrschenden Gegebenheiten von langjährigen Windmessungen ableiten. Wie Abbildung 1 zeigt (nach Messungen des Israel Meteorological Service aus den Jahren 1960 bis 1969; vgl. dagegen die älteren Daten von FrIEDMAN 1969), weht der Wind zu über $70 \%$ aus nördlichen Richtungen (hauptsächlich N-NNO) bei einer durchschnittlichen Windstärke von 2-4 Beaufort. Das bedeutet, daß die in Ufernähe abgebremsten und sich leicht fächerförmig ausbreitenden Oberflächenwellen überwiegend von NO-ONO gegen die Stuitzpfeiler laufen.

Die Seegangshöhe lag meistens unter $1 / 2 \mathrm{~m}$; nur an durchschnittlich drei bis vier Tagen pro Monat erreichten oder überschritten die Wellen in Uferferne $1 \mathrm{~m}$ Höhe. Wenn die Wellen gegen die Pfeiler laufen, entsteht schon bei 2-3 WS eine "Schwappbewegung" mit Luftblasenuntermischung, die in der obersten Wasserschicht von ungefähr $50 \mathrm{~cm}$ vorwiegend vertikal gerichtet ist. Darunter wurden komplizierte Wasserbewegungsformen als Ergebnis der zunehmenden Überlagerung der Oberflächen- 
bewegung durch die uferparallele Längsströmung festgestellt. Dieser Bereich wird kurz als "Turbulenzzone" bezeichnet (Abb. 2)*.

Unterhalb einer Tiefe von 2-3 $\mathrm{m}$ strich diese Längsströmung (dem Genauigkeitsgrad der angewandten Meßmethode entsprechend) weitgehend laminar an den Stützpfeilern entlang, wobei sie zu rund $80 \%$ aus nördlicher Richtung kam. Zuweilen konnte sie aber auch in die umgekehrte Richtung umschlagen, unabhängig davon, ob die Oberflächenwellen unter dem Einfluß des Windes weiterhin in südliche Richtung getrieben wurden. Um die Gesetzmäßigkeit dieser Strömungsverhältnisse einmal näher erfassen zu können, sind Meßreihen über einen längeren Zeitraum hinweg notwendig. Strömungsgeschwindigkeit und -bahn wurden mit Hilfe flottierender Seidenpapierschnitzel gemessen. Die Geschwindigkeit betrug durchschnittlich $3-6 \mathrm{~cm} / \mathrm{sec}$ bei Spitzengeschwindigkeiten von $10-12 \mathrm{~cm} / \mathrm{sec}$. Diese Werte gelten für den Abschnitt der äußeren vier Pfeilerreihen, dahinter wird die Strömungsgeschwindigkeit bis unter die Hälfte der vor der Außenreihe gemessenen Werte gebremst. Das gleiche gilt auch für den Bereich von 1-2 m über dem Böschungsgrund, wo die Wasserbewegung durch vom Boden aufragende Felsblöcke erheblich gemindert wird. Infolge der relativen Wasserstagnation in diesem Bereich kommt es zu einer dichten Sedimentation von Feinpartikeln und Detritus. Der Sauerstoffgehalt lag von der Oberfläche bis über diesen Stagnationsbereich ungefähr beim Sättigungswert.

Die Hafenstützpfeiler wurden in erster Linie unter dem Aspekt als Lichtorgel untersucht. Dabei wurde der Lichtgradient feiner unterteilt, als für die vorliegende Betrachtung notwendig ist. Die Differenzierung in eine Dunkelzone 0,1-0,5\%, Dämmerzone 0,5-2\% und Hellzone 2-13\% der über der freien Oberfläche gemessenen Helligkeit scheint hier ausreichend zu sein (Abb. 2).

\section{ERGEBNISSE}

\section{Vorkommen von Acabaria pulcbraund A. biserialis auf den Hafenstützpfeilern}

Beide Acabaria-Arten, A. pulcbra und A. biserialis, wurden nur in der Dämmerzone der Pfeiler gefunden. Hier kamen sie aber deutlich getrennt voneinander vor. A. pulchra wurde von der Rückseite des ersten bis zum fünften Pfeiler gefunden (Abb. 2). Sehr häufig war die Art auf dem zweiten bis vierten Pfeiler, wo sie zwischen 20 bis $80 \mathrm{~cm}$ Tiefe die auffälligste Art darstellte und dichte Rasen bildete. Zunehmend lockerer verteilt fanden sich die Kolonien noch bis zu 2,5 m Tiefe. In allen Fällen waren die maximal $6 \mathrm{~cm}$ hohen Kolonien sparrig in alle Richtungen hin verzweigt. Anastomosen zwischen den einzelnen Aisten waren selten (Abb. 4). Neben A. pulchra wurden auch einige wenige Exemplare der äußerlich sehr ähnlichen Art A. erytbraea gefunden. Außerdem waren stellenweise zwischen die Acabaria-Bestände lockere Rasen von Laomedea dichotoma eingesprengt. Letztere Art wurde ebenso wie die beiden ge-

* Bei der Betrachtung der Hafenstïtzpfeiler als Lichtorgel (SchuHMacher 1973) wurden die Strömungsverhältnisse etwas gröber differenziert und der Begriff "Turbulenzzone* beschränkt auf den unmittelbar den Oberfächenwellen unterworfenen Bereich gebraucht. 
nannten Acabaria-Arten nur in diesem Bereich der Pfeiler gefunden. Tagsüber waren die einzelnen Polypenkelche von $A$. pulchra trotz der nur schwachen Beleuchtung überwiegend eingezogen.

Militärische Sicherheitsbestimmungen verboten leider nächtliche Kontrollen der

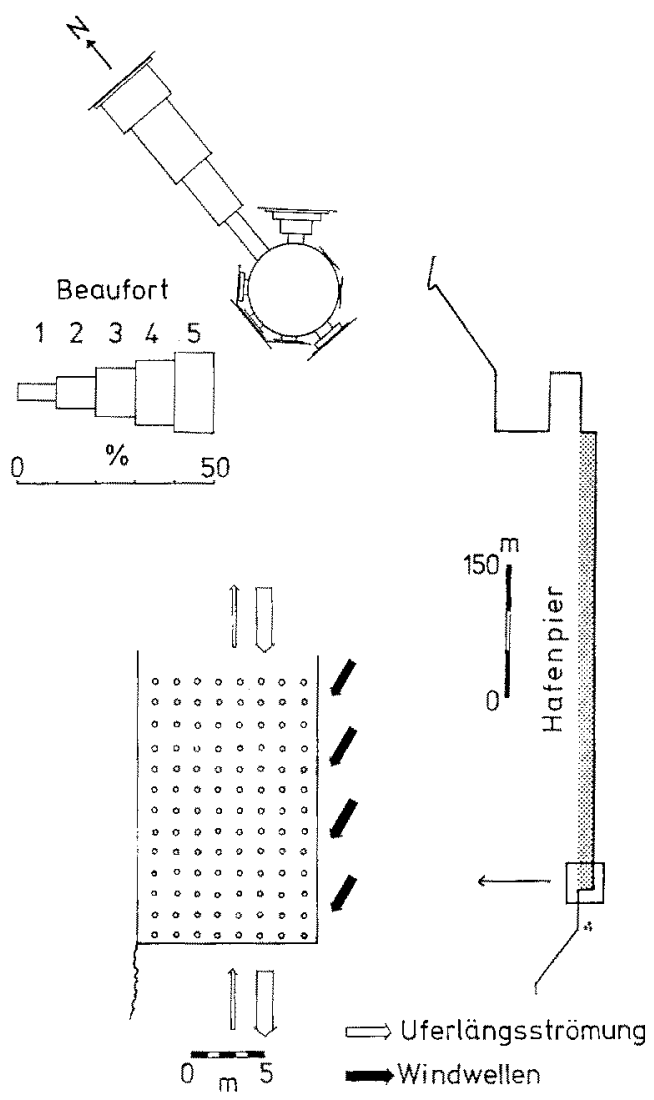

Abb. 1: Lage des Untersuchungsgebietes am Südende der Hafenpier; die Angaben zur Häufigkeit der verschiedenen Windrichtungen und -stärken sind Mittelwerte der Jahre 1960-1969

Pfeiler; bei in ein Aquarium übertragenen Stödken entfalteten sich jedoch die Polypen regelmäßig bei Nacht (Abb. 4).

Acabaria biserialis kam auf der zweiten bis sechsten Pfeilerreihe von $2 \mathrm{~m}$ Tiefe an bis 1 bis $2 \mathrm{~m}$ über Grund vor (Abb. 2). Diese Art bildet keine so dichten Bestände wie A. pulchra, vielmehr stehen die Kolonien einzeln; auf der dritten und vierten Pfeilerreihe (wo diese Art am häufigsten war) wurden Abstände zwischen $30-80 \mathrm{~cm}$ zwischen den einzelnen Stöcken gemessen. Gegen Ende des Untersuchungszeitraums waren diese Bestände leider durch souvenirsammelnde Sporttaucher zunehmend gelichtet worden. Im Vergleich zu der mittel- bis dunkelrot gefärbten Art A. pulchra ist A. biserialis leuchtendgelb oder orange gefärbt. Die einzelnen Kolonien erreichten $15-20 \mathrm{~cm}$ Höhe bei einer Breite von $10-15 \mathrm{~cm}$. 
Sie waren stets flächig in nur einer Ebene entwickelt (Abb. 5). Bezeichnenderweise waren die Stöcke mit kaum einer Ausnahme stets an den Ost- bzw. Westseiten der Pfeiler angesiedelt und ragten von hier als senkrecht stehende Filterfächer in die gleichmäßig aus nördlicher Richtung (oder selten von Süden) vorbeistreichende Längsströmung. Die Polypen waren auch bei dieser Art bei Tag überwiegend eingezogen.

Das deutlich getrennte Vorkommen der beiden Arten an den Hafenpfeilern konnte nur durch die unterschiedliche Art der Wasserbewegung erklärt werden, nach-

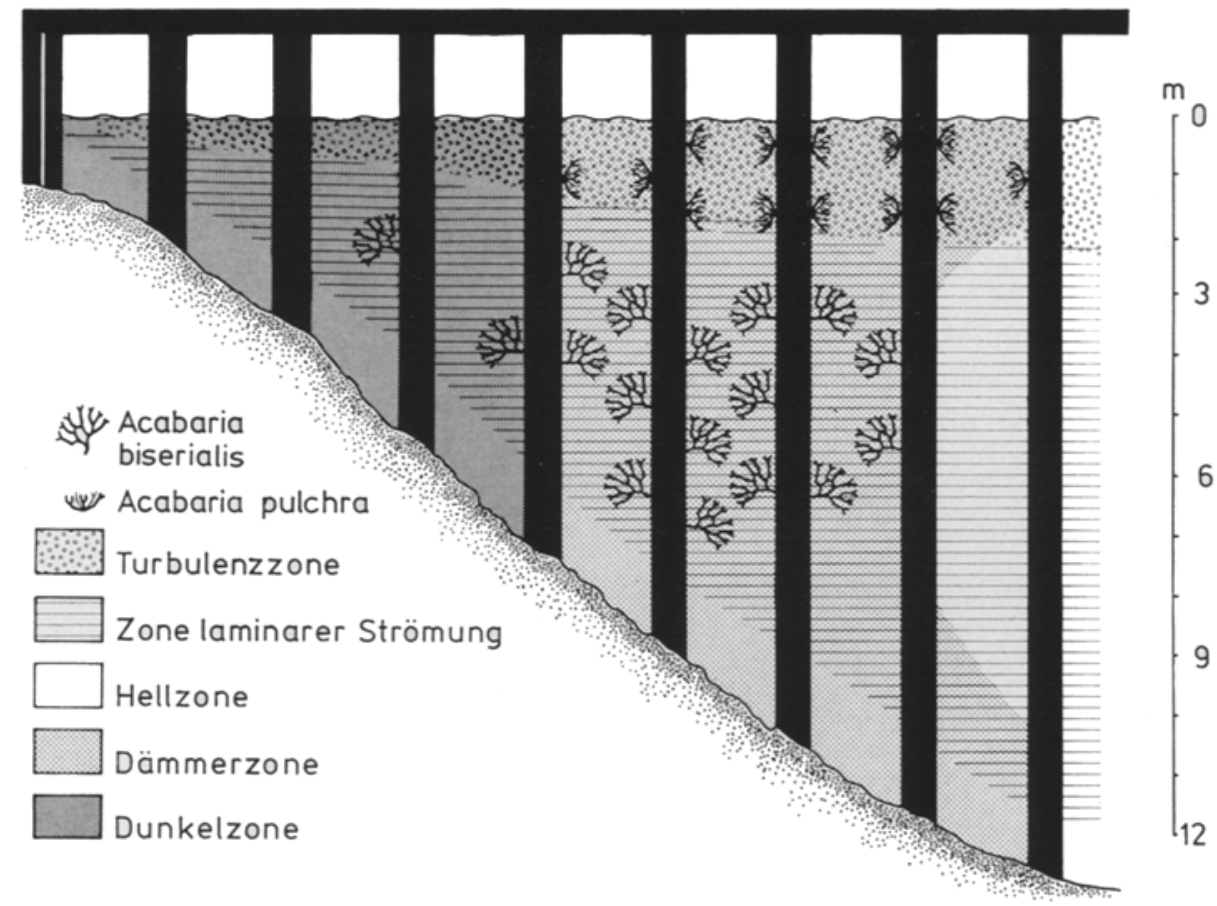

Abb. 2: Schematischer Querschnitt der Hafenpier mit Stützpfeilern. - Verteilung von Acabaria pulchra und $A$. biserialis in Abhängigkeit von Licht und Wasserbewegung

dem an den verschiedenen Ansiedlungspunkten ungefähr gleiche Helligkeitswerte und gleicher Sauerstoffgehalt festzustellen waren.

\section{Vorkommen von Acabaria pulcbra und $A$. biserialis im Korallenriff}

Bei Tauchgängen in Korallenriffen bei Eilat wie auch weiter südlich an der Sinaiküste (Ras el Bourka, Dahab) oder bei Aqaba wurde besonders auf das Vorkommen der beiden Arten Acabaria pulchra und A. biserialis geachtet. Es zeigte sich dabei, daß A. pulchra die einzige Gorgonarie ist, die schon auf dem Riffdach vorkommt, wo als 


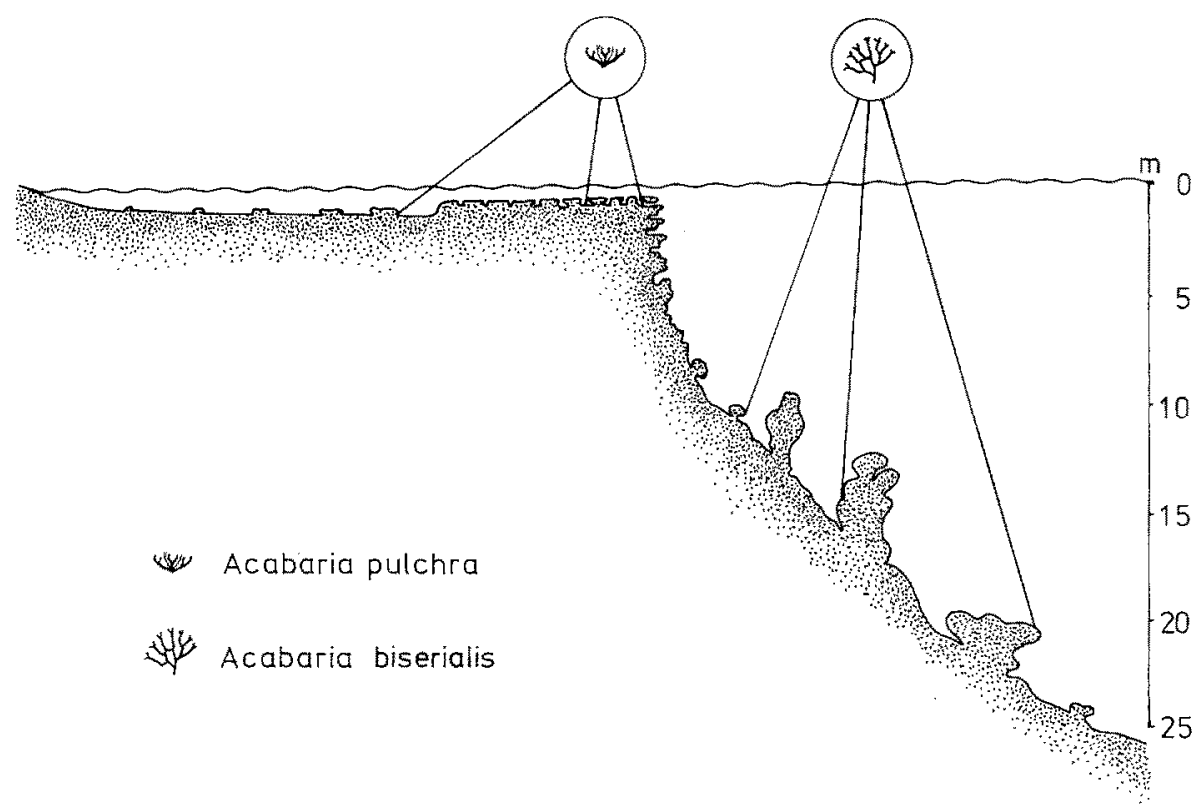

Abb. 3: Schematische Ubersicht über das Vorkommen von Acabaria pulchra und A. biserialis im Riff

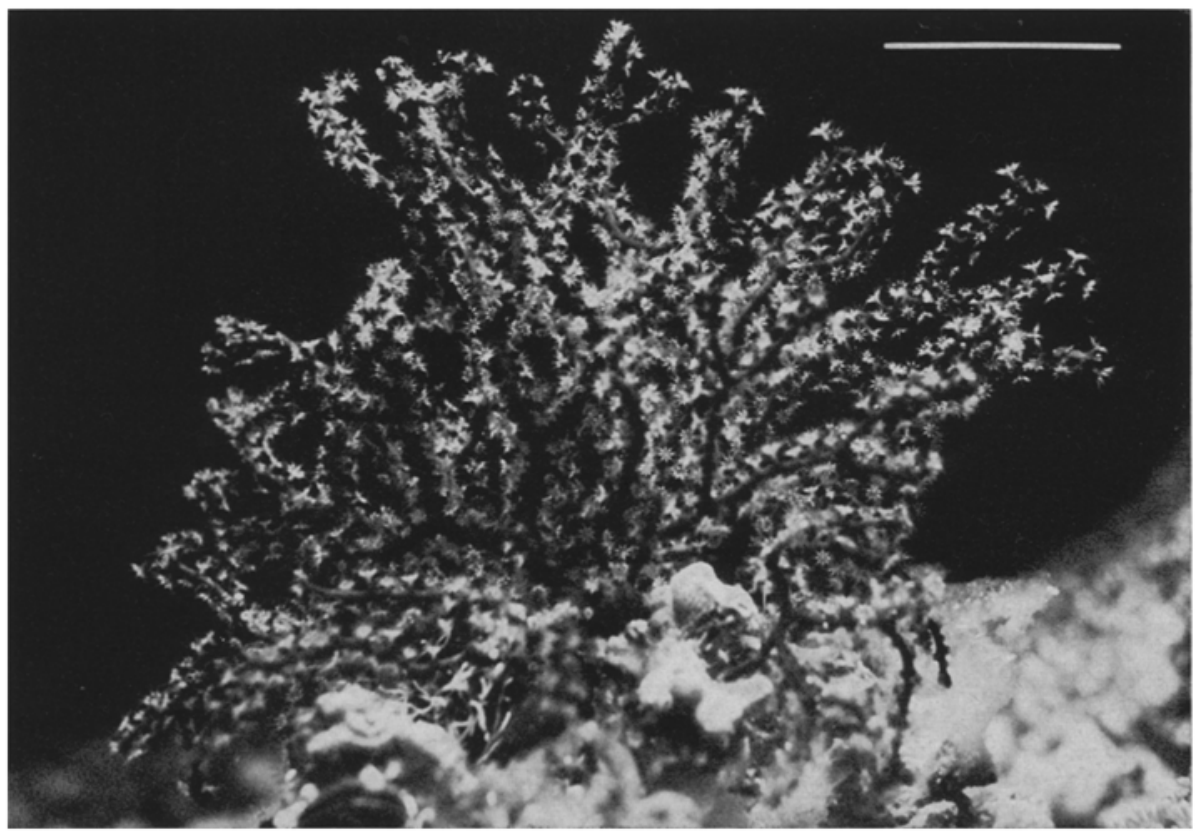

Abb. 4: Acabaria pulcbra mit nächtlich ausgestreckten Polypen (Aquarienaufnahme einet von Hafenpfeilern stammenden Kolonie); $\Leftrightarrow 2 \mathrm{~cm}$ 
weitere Vertreter der Oktokorallen dagegen Alcyonarien wie z. B. Litopbyton arboreum oder Sinularia- und Lobopbytum-Arten geradezu aspektbestimmend auftreten.

Dank dieser leichten Zugänglichkeit gehört die Art zu den häufigsten Gorgonarien-Nachweisen rom Roten Meer, insbesondere wenn man einen Teil angeblicher

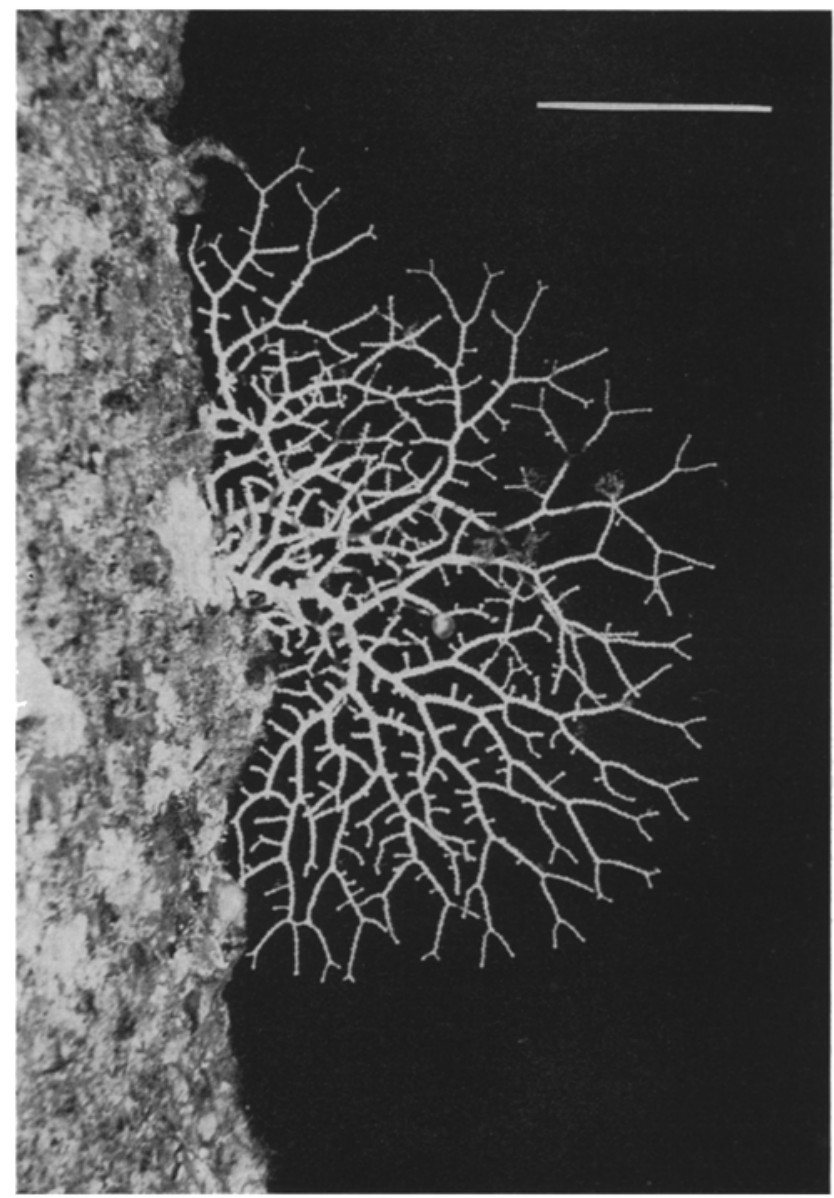

Abb. 5: Kolonie von Acabaria biserialis an einem Hafenstützpeiler;

$\leq 4 \mathrm{~cm}$

A.-erytbraea-Funde miteinbezieht, die nach HICkson (1940) als fehlbestimmte A. pulchra anzusehen sind. Schon KLUNZINGER (1877) gibt als Fundort (von Mopsea erythraea - nach Hickson identisch mit $A$. pulchra) an: „häufig oben am Korallenabhang, besonders zwischen Steinen und in Höhlungen desselben, ist also kein Tiefenbewohner". Auch die von Crossland bei Al Ghardaqa und anläßlich der Mabahith-Expedition gesammelten Stücke stammten nach STIASNY (1940) vom flachen Riffdach. DEAN (1929) berichtet von einer Kolonie, welche als Aufwuchs an einem in flachem Wasser bei Port Said gesunkenen Schiff gefunden worden war. 
Grundsätzlich stimmen alle diese Nachweise mit den eigenen Befunden überein, wonach die Art nur auf dem äußeren Riffdach angetroffen wurde. Regelmäßig findet sich Acabaria pulchra in dem Grotten- und Höhlensystem, wie es für den Bereich der Riffkante typisch ist (Abb. 3). In diesen unregelmäßig verzweigten Kanälen herrschen mit jeder auf- und ablaufenden Brandungswelle wechselnde, auf jeden Fall aber turbulente Wasserbewegungen vor. Der Sauerstoffgehalt ist sehr hoch, of sind feine Luftbläschen untergemischt. Abgesehen von den Kanalöffnungen ist es in diesem Grottensystem dämmrig bis dunkel. Weiter uferwärts wurde die Art zuweilen unter größeren, hohl liegenden Korallenbruchstücken oder losen Tridacna-Schalen gefunden. Diese Kolonien waren jedoch selten über $2 \mathrm{~cm}$ groß und kaum verzweigt.

Acabaria biserialis wurde $u$. a. bei Dredgefängen der Pola-Expedition aus größerer Tiefe gesammelt (Hickson 1940). Bei eigenen Tauchabstiegen wurde A. biserialis am tieferen Riffhang (unter $7 \mathrm{~m}$ ) oder im Vorriff gefunden (Abb. 3). Auch hier ragten - entsprechend den Beobachtungen an den Hafenpfeilern - die Fächer quer zur Rifflängsströmung in den freien Wasserraum. Die Kolonien waren z. T. bei einer Ausdehnung von $20 \times 30 \mathrm{~cm}$ erheblich größer als die an den Pfeilern gefundenen Exemplare.

\section{DISKUSSION}

Wenn auch die Wasserbewegung in ihrer Bedeutung als ökologischer Faktor erst in jüngerer Zeit vermehrt Gegenstand detaillierter Untersuchungen geworden ist, so ist die Ausrichtung festsitzender wie mobiler planktonfiltrierender Organismen (wie z. B. Hydroiden oder Echinodermen) nach der Wasserströmung schon seit langem bekannt und einleuchtend. An Stelle von Literaturhinweisen zu diesem weiteren Problemkreis sei hier nur auf RIEDL (1971) verwiesen, der hierzu eine umfangreiche Übersicht gegeben hat.

Hinsichtlich der planktonfangenden Gorgonarien vermutete schon unter anderen KüKENTHAL (1925), daß sich die fächerartigen Kolonien quer zur vorherrschenden Wasserströmung ausrichteten. WAINWRIGHT \& DiLLON (1969) konnten diese Stellung aus Gesichtspunkten mechanischer Beanspruchung begründen. Die jüngste experimentelle Bestätigung hierzu lieferte Velrmirov (1973) bei Eunicella cavolinii.

ABEL (1959) vermutete auf Grund seiner Untersuchungen an Eunicella-Arten des Mittelmeeres, daß neben der Wasserbewegung als verbreitungs- und formbestimmender Faktor auch das Licht einen sich gleichermaßen äußernden Einfluß hat. Schon dieses eine Beispiel belegt die eingangs aufgezeigte Schwierigkeit, Rückschlüsse auf die am jeweiligen Standort herrschenden Umweltbedingungen zu ziehen, sofern es sich nicht um eine "definierte" und kontrollierte Versuchsanordnung handelt. Diese Unsicherheit bleibt auch bestehen, wenn zu obigem Beispiel nachzutragen ist, daß sich ABEL (mündliche Mitteilung) später von seiner Lichthypothese etwas distanziert hat (nach RIEDL 1964).

Dank der günstigen Situation der Versuchsanlage Hafenstützpfeiler konnten beide Faktoren Licht und Wasserbewegung in ihrem Einfluß nebeneinander kontrolliert werden. Nachdem die Stützpfeiler schon über sechs Jahre im Wasser standen, war die vorgefundene Artenverteilung sicher keine vorübergehende Zufallserscheinung. Es kann 
vielmehr als sicher gelten, daß jede Art genau das Areal besiedelte, welches am ehesten der spezifischen ökologischen Nische entsprach. Bei den Arten Acabaria pulcbra und A. biserialis bestanden hinsichtlich der Helligkeit ungefähr die gleichen Ansprüche: Dämmerlicht von 2-0,5\% der über der freien Wasserfläche gemessenen Beleuchtungsstärke.

Dagegen zeigen die beiden Arten unterschiedliche Ansprüche und dementsprechend verschiedene morphologische Anpassungen an die Wasserbewegung.

A. pulchra fand sich auf den Pfeilern fast ausschließlich nur im Einflußbereich der anlaufenden Wellen - in einer Zone mit sehr unregelmäßig verlaufenden Strömungen. Entsprechend ungerichtet entwickelten sich die Kolonien strauchförmig in alle Raumrichtungen. Im Vergleich zu $A$. biserialis oder anderen fächerartig wachsenden Gorgonarien blieben die Kolonien niedrig; auch an der dritten oder vierten Pfeilerreihe, wo der Strömungsdruck der sich brechenden Wellen wesentlich geringer als an der Außenreihe war, erreichten die Stöcke höchstens eine Höhe von $6 \mathrm{~cm}$. Bei dieser Größe wurden sie auch bei heftigem Wellengang nicht abgebogen.

Der sparrige, niedrige Wuchs scheint eine arttypische Anpassung an die Strömungsverhältnisse im Brandungsbereich des Riffs zu sein, wo laufend wechselnde Fließrichtungen und zeitweilig hohe Strömungsdrucke auftreten. Bei dieser Art sind die Polypen gleichmäßig um die Zweige herum verteilt. In einer Kollektion von 50 wohlerhaltenen A.-pulchra-Kolonien (aus der auch der Typus der Art stammt), fand HicksoN (1937) nur wenige junge Stöcke, die zweidimensional ausgerichtet waren. Eine gleichermaßen buschig-niedrige Kolonieform zeigt auch die südafrikanische Art $A$. capensis, die bezeichnenderweise mehrfach an Bootsstegen dicht unter der Wasseroberfläche gefunden wurde (Hickson 1937).

Zu der Frage, warum $A$. pulchra nicht auch den tieferen, gleichmäßig beströmten Bereich der Hafenpfeiler besiedelt hat, kann man nur vermuten, daß möglicherweise die Kolonien zu klein und die Polypen zu ungeordnet stehen, um bei verminderter Strömung noch eine ausreichende Wassermenge zu filtrieren. Offensichtlich fehlt bei dieser Art die Potenz zur Vergrößerung bzw. großflächigen Ausrichtung der Kolonie, um den geringeren Wasserdurchsatz im Vergleich zur oberflächennahen Schicht entsprechend zu kompensieren. Eine Abhängigkeit vom Sauerstoffgehalt erscheint unwahrscheinlich, da keine merkbaren Unterschiede gemessen werden konnten. Eine experimentelle Verpflanzung von Acabaria-pulcbra-Kolonien in den Bereich von $A$. biserialis und umgekehrt wurde bisher noch nicht gemacht; dieser Kontrollversuch ist an den Hafenpfeilern aber bei nächster Gelegenheit vorgesehen.

Acabaria erytbraea ist $A$. pulcbra äußerlich zum Verwechseln ähnlich. Während jedoch $A$. pulchra bisher ausschließlich als Flachwasserform bekannt ist, berichtet HrckSON (1940) von größeren fächerartig entwickelten Kolonien, die von der J.-MurrayExpedition an der arabischen Südküste in $40 \mathrm{~m}$ Tiefe gedredgt wurden und mit hoher Wahrscheinlichkeit der Art A. erythraea zuzurechnen sind. Möglicherweise gibt es bei dieser Art je nach Standort eine strauch- und fächerförmige Modifikation.

Acabaria biserialis siedelt sich im Bereich einer gleichmäßig aus einer Richtung kommenden Wasserströmung an, wobei die flächig ausgebildeten Kolonien senkrecht zur Strömung wachsen. Wie der Artname biserialis schon ausdrückt, reihen sich die Polypen entlang der Seiten der Zweige, um den vorbeistreichenden Wasserstrom opti- 
mal auszunutzen. Analoge Beobachtungen an Gorgonia spec. liegen von RIEDL \& FORSTNER (1968) vor. Da bei dieser Stellung der Kolonien ein beträchtlicher Strömungswiderstand gegeben ist, beschränkt sich das Vorkommen der Acabaria-biserialis-Fächer auf Zonen langsamer Strömungsgeschwindigkeiten. Entsprechend wurde die Art im Riff nur an Stellen gefunden, wo bei stark gedämpften Helligkeitswerten die geschilderten Strömungsverhältnisse gegeben waren. Bei Dahab an der Sinaiküste wurden in $40 \mathrm{~m}$ Tiefe Gorgonarienfächer von 1,5-2 m Durchmesser und armdicken Ästen gesehen. Leider war eine Bestimmung der Art bisher noch nicht möglich. Die streng flächige Ausbildung und Stellung der Kolonien quer zur Rifflängsströmung stimmen jedoch mit den Befunden an A. biserialis iberein.

Von den Arten $A$. pulchra und A. biserialis sind bisher keine verschiedenen Standortmodifikationen bekannt. Die Beobachtungen im Riff und die eindeutigen Feststellungen an den Hafenstützpfeilern legen vielmehr die Annahme nahe, daß die unterschiedlichen Wuchsformen arttypisch sind und sich die Arten im Vorkommen gegenseitig ausschließen.

Die Ausbildung der Kolonien von Acabaria biserialis entspricht den typischen "sea fans", wie sie sich in Bereichen mäßiger, gleichgerichteter Strömungen an allen Meereskïsten finden. Daß diese Strömungsverhältnisse tatsächlich eine, wenn nicht gar die entscheidende Voraussetzung für einen "Gorgonarien-Biotop" darstellen, zeigt das Beispiel der Hafenpfeiler, wo in dem von A. biserialis besiedelten Bereich noch die Gorgonarien Clatbraria rubrinodis, Acanthogorgia spec. und Suberogorgia spec. vorkamen.

Zumindest im Roten Meer hat nur Acabaria piulchra (zusammen mit A. erytbraea) einen Lebensraum erschlossen, der mit seinen wechselnden Strömungsverhältnissen zunächst gar nicht für einen immobilen, starren Planktonfiltrierer geeignet erscheint. Die allseitig sparrig verzweigte Kolonieform sowie die gleichförmig verstreute Anordnung der Polypen stellen aber offensichtlich ausreichende Anpassungen dar, um unter diesen abweichenden Lebensbedingungen das Fortkommen zu sichern.

\section{ZUSAMMENFASSUNG}

1. Da standort- und formbestimmende Einflüsse ökologischer Faktoren auf Organismen im ausgebildeten Korallenriff meist nur sehr unzulänglich bestimmbar sind, wurde die Wirkung verschiedener physikalischer Einflüsse an speziell hierfür geeigneten Kunstbauten bei Eilat (Israel) im Golf von Aqaba untersucht. So wurde der Einfluß der Wasserbewegung an Gorgonarien studiert, die auf den Stützpfeilern im Handelshafen von Eilat siedelten.

2. Die beiden Arten Acabaria pulchra und A. biserialis kommen im gleichen Helligkeitsbereich $(2-0,5 \%$ der über der freien Wasserfläche gemessenen Beleuchtungsstärke) vor. $A$. pulchra ist auf den Turbulenzbereich der anlaufenden Wellen in den obersten $2 \mathrm{~m}$ Wassertiefe beschränkt. Die Strömungsverhältnisse sind bier durch unterschiedlich gerichtete Wasserbewegung und ständig wechselnden Strömungsdruck charakterisiert. Dagegen kommt $A$. biserialis im Bereich langsamer bis mäßig starker $(3-12 \mathrm{~cm} / \mathrm{sec})$, vor allem aber stets gleichgerichteter Strömung vor. Diese Verhältnisse waren im Bereich der Hafenpfeiler in einer Tiefe von ca. 2-6 m gegeben. 
3. Bei Vergleichsbeobachtungen an verschiedenen natürlichen Riffen des Golfs von Aqaba fand sich $A$. pulchra unter den typischen Bewohnern des Grotten- und Höhlensytems der Riffkante. A. biserialis wurde fast ausschließlich im Dämmerbereich unter $7 \mathrm{~m}$ Tiefe im Bereich der Rifflängsströmung gefunden. Durch die ständig anund ablaufenden Brandungswellen ist dieser Bereich fortwährend wechselnden Strömungsverhältnissen ausgesetzt.

4. Die Kolonieform scheint arttypisch den verschiedenen Strömungsbedingungen zu entsprechen: $A$. pulchra wächst sparrig strauchförmig in alle Richtungen. Die Kolonien bleiben niedrig $(6 \mathrm{~cm})$ und werden auch bei starkem Strömungsdruck nicht abgebogen. Die Polypen sind gleichmäßig rings um die Zweige angeordnet. A. biserialis entwickelt sich flächig und dabei quer zur vorherrschenden Strömungsrichtung, um einen maximalen Filtriereffekt zu erzielen. Die fächerartigen Kolonien erreichen eine Höhe von $30 \mathrm{~cm}$. Die Polypen sind zweireihig längs der Seiten der Zweige angeordnet.

5. Acabaria pulchra ist neben der habituell gleich erscheinenden, aber seltenen $A$. erytbraea die einzige Gorgonarie im Roten Meer, die in den Bereich der Riffkante mit seinen wechselnden Strömungsrichtungen und -drücken vorgedrungen ist. Als Anpassungen an die Verhältnisse werden der niedrige Wuchs, die allseitig sparrig verzweigte Form und die gleichmäßige Verteilung der Polypen auf den Zweigen angesehen.

Danksagungen. Herrn Dr. M. Grasshoff (Senckenberg-Museum, Frankfurt/M.) danke ich sehr für die Bestimmung der taxonomisch schwierigen Gorgonarien. Den Mitgliedern des Marine Biological Laboratory in Eilat und dessen inzwischen verstorbenen Leiter, Dr. H. STEINITZ, danke ich für die erwiesene Unterstützung während meines Aufenthaltes an der Station. Die Untersuchungen wurden in dankenswerter Weise von der Deutschen Forschungsgemeinschaft durch Bereitstellung eines Forschungsstipendiums ermöglicht.

\section{ZITIERTE LITERATUR}

ABEL, E. F., 1959. Zur Kenntnis der marinen Höhlenfauna unter besonderer Berücksichtigung der Anthozoen. Ergebnisse der österreichischen Tyrrhenia-Expedition 1952, Teil 5. Pubbl. Staz. zool. Napoli (Suppl.) 30, 1-94.

DEAN, L. M. I., 1929. Report on the Alcyonaria, Zoological results of the Cambridge Expedition to the Suez Canal, 1924. Trans. zool. Soc. Lond. 22, 707-712.

FrIEDMAN, G. M., 1968. Geology and geochemistry of reefs, carbonate sediments, and waters. Gulf of Aqaba (Elat), Red Sea. J. sedim. Petrol. 38, 895-919.

Hrckson, S. J., 1937. The family Melitodidae. Trans. zool. Soc. Lond. 23, 73-212.

- 1940: The species of the genus Acabaria in the Red Sea. Publs. mar. biol. Stn. Ghardaqa 2, $3-22$.

Klunztnger, C. B., 1877. Die Korallenthiere des rothen Meeres. 1. Die Alcyonarien und Malacodermen. Gutmann, Berlin, 98 pp.

KüKenTHAL, W., 1925. Octocorallia. Handb. Zool. 1, 690-769.

RIEDL, R., 1964. 100 Jahre Litoralgliederung seit Josef Lorenz, neue und vergessene Gesichtspunkte. Int. Revue ges. Hydrobiol, 49, 281-305.

- 1971. Water movement: animals. In: Marine ecology. Ed. by O. KrNNE. Wiley-Interscience, London 1 (2), 1123-1156.

- \& Forstner, H., 1968. Wasserbewegung im Mikrobereich des Benthos. Sarsia 34, 163-188. 
Schummacher, H., 1973. Die lichtabhängige Besiedlung von Hafenstützpfeilern durch sessile Tiere und Algen aus dem Korallenriff bei Eilat (Rotes Meer). Helgoländer wiss. Meeresunters. 24, 307-326.

STODDART, D. R., 1969. Ecology and morphology of recent coral reefs. Biol. Rev. 44, 433-498.

Velrmirov, B., 1973. The orientation of Ennicella cavolinii (sea fan) related to water movement. Helgoländer wiss. Meeresunters. 24, 163-173.

Wainwright, S. A. \& Dillon, J. R., 1969. On the orientation of sea fans (genus Gorgonia). Biol. Bull. mar. biol. Lab., Woods Hole 136, 130-140.

Anschrift des Autors: Dr. H. Schummacher

Ruhr-Universität Bodhum

Lehrstuhl für Spezielle Zoologie

463 Bochum-Querenburg

Bundesrepublik Deutschland 